\title{
Industrialization and Manufacturing Steps within the Global Product Lifecycle Context
}

\author{
Anis Ben Khedher ${ }^{1}$, Sébastien Henry ${ }^{2}$, and Abelaziz Bouras ${ }^{1}$ \\ ${ }^{1}$ Université de Lyon, Lumière Lyon 2, IUT Lumière, \\ LIESP Laboratory, Bron, 69676, France \\ \{anis.ben-khedher, abdelaziz.bouras\}@univ-lyon2. fr \\ ${ }^{2}$ Université de Lyon, Claude Bernard Lyon 1, IUT B, \\ LIESP Laboratory, Villeurbanne, 69622, France \\ sebastien.henry@univ-lyon1.fr
}

\begin{abstract}
This paper presents and discusses an analysis of the industrialization and production steps within the Product Life cycle Management (PLM) context. Initially, PLM was focused almost exclusively on the product design, but nowadays, it tends to cover all the steps of the product life cycle. In the same time, the industrialization and the production are not sufficiently integrated into the PLM solutions. Currently, there is much to be gained by extending the coverage of PLM to production step. This coverage depends on several features (for instance the frequency of product data modification). It also leads to an information exchange then to a classification of this information into categories. The main purpose of this paper is to study how to extend the PLM coverage of the life cycle steps by defining a mapping among information categories and the Information Systems (IS), which manages product manufacturing, for each feature.
\end{abstract}

Keywords: Product Lifecycle Management, production management, information systems interoperability.

\section{Introduction}

Manufacturing enterprises are facing several challenges such as shorten innovation lead-times, reduction of time to market, reduction of costs, mass customization demands, more complex products, improving product quality, geographically dispersed design teams, inventories subject to rapid depreciation and rapid fulfillment needs [1]. Nowadays, the need of deploying PLM system becomes more and more important in order to tackle theses challenges. The PLM is a business strategic approach that leads to develop an attractive system which ensures customer satisfaction. PLM applies a set of business solutions to support the collaborative creation, management, dissemination, and the use of product definition information across the extended enterprise from concept to end-of-life. It integrates people, processes, business systems and information together [2]. The product lifecycle consists of three main phases: the Beginning Of Life (BOL) including requirements, design, industrialization and production; the Middle Of Life (MOL) including logistics, use, and maintenance; and 
End Of Life (EOL) including reverse logistics, remanufacturing, reuse, recycle, and disposal [3]. In fact, with the increasingly demand of complex products and the advancements in Computer Aided Design (CAD), more and more data are being generated. These data are related to the development of products such as CAD drawings, 3D models and the documentation related to the requirements management and development [4]. Currently, design consists of the most developed product life cycle step. Thus, PLM covers requirements analysis, design and industrialization almost total way. In fact, even if it covers all life cycle phases, there are still many interactions not yet resolved such as the interaction with production management. Therefore, PLM focuses mainly on virtual product and process development. The interaction with production management becomes more and more required. The coverage of production management by PLM leads to the full interaction between the two parties. It is dependent on several features such as frequency of product data modification, number of manufacturing systems producing the same product and so on. The definition of these features and its impact on the PLM coverage is also very important to avoid data inconsistencies. The identification of exchanged information between PLM and production management is also important. PLM should support the communication of information to production and consequently information feedback [5]. In order to accomplish the study of the PLM coverage extension, we propose to identify the different exchanged information between PLM and production management and classify this information into categories such as product design, manufacturing process, manufacturing system configuration and manufacturing system design. After defining the features and information categories, we suggest to identify the different IS managing production such as Manufacturing Execution System (MES) and Enterprise Resource Planning (ERP). Therefore, we can establish a mapping among information categories and IS that allows us to define what information can be managed by what IS according to the features defined earlier. This paper describes the PLM coverage of life cycle steps, particularly the production step. We first present the features that impact this coverage and the information categories that can be exchanged between PLM and production management. The mapping among information categories and IS according to the features is also presented before the conclusion.

\section{Closed-Loop PLM}

During BOL, the information flow is quite complete between design and industrialization. Virtual product and process data such as product design, Electronic Bill Of Material (EBOM), Manufacturing Bill Of Material (MBOM), process plans and so on; are produced within design and industrialization product lifecycle steps. However, the information flow becomes vague or unrecognized after the industrialization step. This prevents the feedback of product-related information such as production status, updated $\mathrm{BOM}$, updated process plans and so on; from production back to engineering [3]. Both design and industrialization steps form the engineering phase within BOL. Sufficient and up-to-date engineering data should be communicated to production management in order to start the product manufacturing. In return, design and industrialization need sufficient and dynamic real-time shop floor data gathered by production to optimize and enhance its engineering data and works. These feedbacks of 
product information become important in order to allow each lifecycle step to make decisions while having visibility to others lifecycle steps.

To synthesize, we conclude that this data exchange is between high-level information provided by design and industrialization and low-level information provided by production management. It allows forming a closed loop among design, industrialization and production as shown in Fig.1.

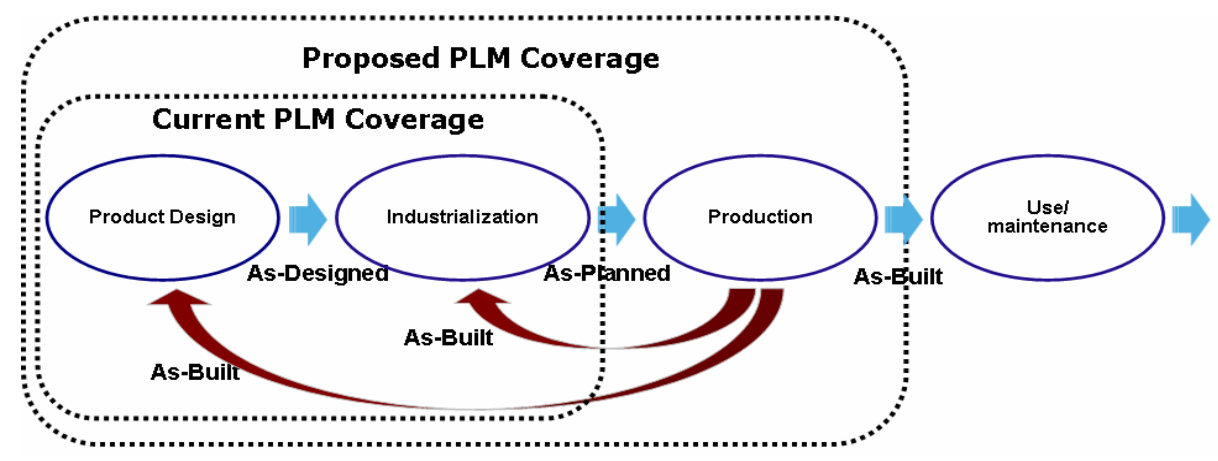

Fig. 1. Closed loop among design, industrialization and production

\section{Problem Statement}

In an extended enterprise context, PLM support needs to connect the product design and analysis processes to the production and vice versa [5]. In order to realize this goal, PLM should cover design, industrialization and production steps. The coverage of the different steps may vary according to several features. For instance we can identify:

- Frequency of product data modification. When this frequency is high that means that the information flow that begins from design in coming to production become important. This increase of information flow is due to the product-related information modification such as requirements, designs, BOMs and so on. This flow of information is low when the enterprise produces the same products without modifications so the frequency of product data modification becomes low.

- Frequency of making to market of new products. This frequency is high when the enterprise introduces sometimes new products. The creation, development and introduction of this new product imply the creation of new designs, BOMs, process plans and so on. Therefore, the information flow from design to production increases. However this flow of information is low when the enterprise produces the same products for long periods of time so the frequency of making to market of new products becomes low.

- Number of manufacturing systems producing the same product. We distinguish two cases: In the first case, the enterprise has different manufacturing systems located in several countries implying a difference in ways and 
means to produce the same product. For example, the cost of labor is low in Asia that promotes manual production. However, in other countries the same product is manufactured by machines and robots. In this case, the coverage of product life cycle steps by PLM cannot exceed the common process plan produced during the industrialization step. Preserving the specificity of each manufacturing site becomes important. In the second case, we have the same manufacturing systems that produce the same product.

To synthesize, the PLM coverage of product life cycle steps should be variable according to these features.

\section{Exchanged Information among Design, Industrialization and Production}

As we know, the interaction between design, industrialization as a high-level information and production management as low-level information becomes important. Therefore, it seems necessary to classify this information into categories. In fact, we distinguish four categories of product-related information: product design, manufacturing process, manufacturing system configuration and manufacturing system design. Exchanged information among design, industrialization and production is shown in Fig.2.

\subsection{Information Required for Production}

The most important advantage of data exchange between PLM and production management is to allow production enforcing as-planned record generated by industrialization step and also enforcing manufacturing system configuration data and manufacturing system design data as shown in Fig. 2. All these data should be communicated to production. The as-planned record include the product information provided by $\mathrm{CAD}$ tools, such as the attributes of the parts, product design and drawing, structure relationship, EBOM and so on; and the manufacturing process information provided by Digital Manufacturing (DM) and Manufacturing Process Management (MPM) tools, such as process plans, manufacturing proceeding, 3D models, MBOM and so on [6]. The data generated by manufacturing system configuration determine how to produce the product such as routes, work instructions, man-hour, and Programmable Logic Controllers (PLC) code, Compute Numerical Control (CNC) code, manufacturing system parameters, Human Machine Interface (HMI) code and things alike [7]. The manufacturing system design is the step that contains information related on the capacity of manufacturing system to produce the appropriate product. It communicates to production all information about machines notice, materials, machines 3D models, manufacturing system simulations and so on. As we mentioned earlier, all these data should be put into production management in order to manufacture the product using updated engineering data.

\subsection{Information Generated by Production}

Production management is also critical to engineering when it monitors and reports what currently occurred on the physical factory floor. As-built record is the creation 
of a virtual counterpart to the physical product [8]. In fact, the as-built record contains three categories of data: data concern manufacturing process, manufacturing system configuration and manufacturing system design. The data concerning process should be the performance indicators of each operation (non-quality and traceability) and so on; this information is useful to update product and process. In order to update the manufacturing system configuration, the data communicated by production should be the PLC \& CNC program failures and performance indicators and so on. Finally, data concerning manufacturing system design should be the maintenance operations (curative \& preventive), failures and so on. This information are useful modify the physical part of the manufacturing system.

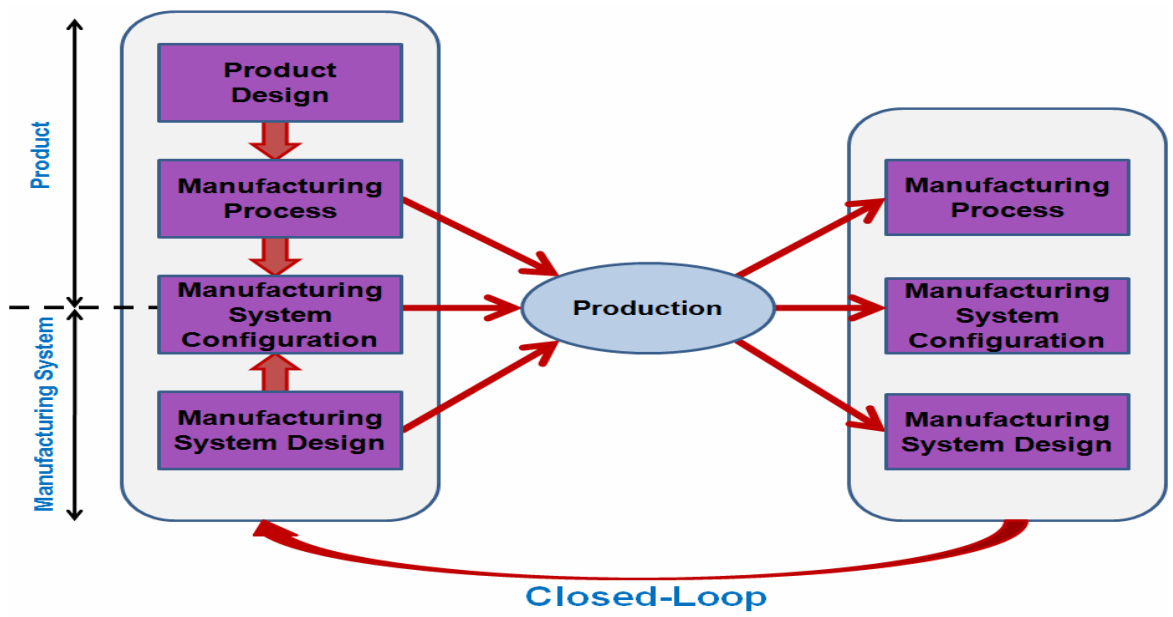

Fig. 2. Exchanged information among design, industrialization and production

\subsection{Closed Loop}

The described information mentioned earlier form the main contents of the data exchange among design, industrialization and production. The transfer of information from production to design/industrialization forms a closed loop among the information categories generated by production and the information categories provided to production. To synthesize, we conclude that this information exchange allows closing the loop from as-built records to as-designed/as-planned records. Fig.2 shows the closed loop formed by design/industrialization and production exchange.

\section{Mapping among Information Categories and ISs}

\subsection{Industrial Information Systems}

In order to lead production operations in the manufacturing enterprise, we distinguish three main IS that manage production. These IS have different tasks and structures. The first system is the PLM that is mentioned earlier. The second is the Enterprise Resource Planning that encompasses a wide range of software products supporting 
day-to-day business operations [9]. It ensures production process optimization control, optimization operation and optimization management technology that takes production synthesis index as the target [10]. Finally, the Manufacturing Execution System that aims at executing manufacturing plans. It builds a bridge between CNC machine tools and PLCs on the lower level and the ERP system on the higher side [11]. MES has eleven functions, defined by the Manufacturing Execution System Association (MESA), such as resource allocation and status, detailed scheduling, dispatching production units, document control, data and so on [12].

\subsection{Mapping Problem}

After identifying the different categories of product-related information as well as the different ISs, we have to define which category of information is managed by which IS. Therefore, we suggest defining a mapping among information categories and IS. The coverage of each of the IS varies according to the features mentioned in section 3. We have chosen to realize tables that summarize this mapping according to each feature. In our study, we focus on the case of companies that have the three IS detailed in section 5.1. Indeed, part of the role of the ERP the product-related information that ERP have are limited to items references and manufacture operations sequence without details. For these reasons, the mapping will focus only on the PLM, MES and other possible systems that can manage product information.

\subsubsection{Mapping According to the Feature: Frequency of Product Data Modification}

When the frequency of product data modification is high, it means that the enterprise produces a customized product. This type of production is characterized by a low volume of production and a high variance of product. Therefore, the enterprise tends to encompass all information categories in PLM system in order to avoid losing product-related information that change rapidly. This is the most ideal mapping that the enterprise can deploy. The MES system is only a user of information. Table 1 shows the mapping according to high frequency of product data modification.

Table 1. Mapping of a high frequency of product data modification

\begin{tabular}{|l|c|c|c|}
\hline & PLM & MES & Other \\
\hline Product Design & $\checkmark$ & & \\
\hline Manufacturing Process & $\checkmark$ & & \\
\hline Manufacturing System Configuration & $\checkmark$ & & \\
\hline Manufacturing System Design & $\checkmark$ & & \\
\hline
\end{tabular}

When the frequency of product data modification is low, it means that the enterprise produces the same product for long periods of time. This type of production is characterized by a high volume of production and a low variance of product. The enterprise tends to encompass product design and manufacturing process in PLM, manufacturing system configuration in MES and manufacturing system design in other IS. The question that arises is why we don't use the ideal mapping in this case. In fact, the consideration of costs and gains is important. The enterprises choose this mapping because its advantage is the information consistency provided by PLM / MES interoperability. Its disadvantage is the PLM/MES interoperability costs. 
Table 2. Mapping of to low frequency of product data modification

\begin{tabular}{|l|c|c|c|}
\hline & PLM & MES & Other \\
\hline Product Design & $\checkmark$ & & \\
\hline Manufacturing Process & $\checkmark$ & & \\
\hline Manufacturing System Configuration & & $\checkmark$ & \\
\hline Manufacturing System Design & & & $\checkmark$ \\
\hline
\end{tabular}

\subsubsection{Mapping According to the Feature: Frequency of Making to Market of New Products}

In fact, the mapping according to the frequency of making to market of new products has almost the same feature as the mapping of high frequency of product data modification. Table 1 and Table 2 show the mapping according to high and low frequency of making to market of new products.

\subsubsection{Mapping According to the Feature: Number of Manufacturing Systems Producing the Same Product}

We suppose in our study that if there are several manufacturing systems that produce the same product then they are necessarily different. Therefore, we distinguish two cases: one production system and several production systems that produce the same product. The first case means that the enterprise doesn't have a management problem of specificities of the different manufacturing systems that produce the same product. Therefore, PLM can encompass all information categories as shown in Table 3.

Table 3. Mapping according to the use of one production system

\begin{tabular}{|l|c|c|c|}
\hline & PLM & MES & Other \\
\hline Product Design & $\checkmark$ & & \\
\hline Manufacturing Process & $\checkmark$ & & \\
\hline Manufacturing System Configuration & $\checkmark$ & & \\
\hline Manufacturing System Design & $\checkmark$ & & \\
\hline
\end{tabular}

When the enterprise deploys several production systems to produce the same product, the task of information management of each manufacturing system becomes difficult. Therefore, the local MES system will manage the manufacturing system configuration and the manufacturing system design. Each local MES system communicates with the global PLM. Table 4 shows the mapping when the enterprise produces the same product by several manufacturing systems.

Table 4. Mapping according to the use of several production systems

\begin{tabular}{|l|c|c|c|}
\hline & PLM & MES & Other \\
\hline Product Design & $\checkmark$ & & \\
\hline Manufacturing Process & $\checkmark$ & & \\
\hline Manufacturing System Configuration & & $\checkmark$ & \\
\hline Manufacturing System Design & & & $\checkmark$ \\
\hline
\end{tabular}




\section{Conclusion}

The need for a more effective methodology supporting the extension of the PLM coverage of life cycle steps is clearly stated by academics and practitioners worldwide. This coverage depends on several features based on information exchanged among design, industrialization and production. The main purpose of this paper is to contribute in extending PLM to production by proposing a mapping among exchanged information and IS which manages the product manufacturing. This mapping is defined for each feature of the PLM coverage. We have suggested three features: frequency of product data modification, frequency of making to market of new products and number of manufacturing systems producing the same product. Besides, we have defined a mapping among information categories and the different IS for each PLM coverage feature which allows us to distinguish the impacts of this mapping on enterprises. This mapping, however, allows us to think about other combinations such as what can be the PLM coverage when the enterprise has several production systems that produce the same product and the frequency of product data modification is high. Therefore, they are important to choose the technical solution that realizes the interaction between PLM and production management, in other words the PLM/MES interoperability. The Service Oriented Architecture (SOA) may be the best architecture [13]. We have chosen this technique because it offers mechanisms of interoperability that allow different technologies to be dynamically integrated, independently of the system's platform in use [14]. Therefore, the services will be adapted to all mapping combinations.

\section{References}

1. Ming, X.G., Yan, J.Q., Wang, X.H., Li, S.N., Lu, W.F., Peng, Q.J., Ma, Y.S.: Collaborative process planning and manufacturing in product lifecycle management. Computers in Industry 59, 154-166 (2008)

2. CIMdata Report, Product Lifecycle Management, Empowering the Future of Business (2002)

3. Jun, H.-B., Kiritsis, D., Xirouchakis, P.: Research issues on closed-loop PLM. Computers in Industry $58,855-868$ (2007)

4. Muhammad, A., Esque, S., Aha, L., Mattila, J., Siuko, M., Vilenius, M., Järvenpää, J., Irving, M., Damiani, C., Semeraro, L.: Combined application of Product Lifecycle and Software Configuration Management systems for ITER remote handling. In: Fusion Engineering and Design (2009)

5. Rachuri, S., Subrahmanian, E., Bouras, A., Fenves, S.J., Foufou, S., Sriram, R.D.: Information sharing and exchange in the context of product lifecycle management: Role of standards. Computer-Aided Design 40, 789-800 (2008)

6. Intercim, Meyer, P., Plapp, J.: Intercim white paper, Extending PLM to the Shop Floor (February 2008)

7. Siemens, Closing the Loop between Engineering and Execution (June 3, 2008), http: / / docs. google. com/gview?a=v\&pid=gmail\&attid=0 . 1\&thid=12 1556dd83bd49bb\&mt=application\%2Fpdf

8. Grieves, M.: Multiplying MES Value with PLM Integration, Whitepaper (March 2007) 
9. Botta-Genoulaz, V., Millet, P.-A.: A classification for better use of ERP systems. Computers in Industry 56, 573-587 (2005)

10. Shaohong, J., Qingjin, M.: Research on MES Architecture and Application for Cement Enterprises. In: 2007 IEEE International Conference on Control and Automation ThB5-5, Guangzhou, China, May 30-June 1 (2007)

11. Iassinovski, S., Artiba, A., Fagnart, C.: SD Builder: A production rules-based tool for online simulation, decision making and discrete process control. Engineering Applications of Artificial Intelligence 21, 406-418 (2008)

12. MES Explained: A High Level Vision, MESA International White Paper Number 6 (September 1997)

13. Izza, S., Vincent, L., Burlat, P.: Ontology-Based Approach for Application Integration. In: Doctoral Symposium, Pre-Proceedings of the First International Conference on Interoperability of Enterprise Software and Applications: INTEROP-ESA 2005, Geneva, Switzerland, February 23-25 (2005)

14. Jardim-Goncalves, R., Grilo, A., Steiger-Garcao, A.: Challenging the interoperability between computers in industry with MDA and SOA. Computers in Industry 57, 679-689 (2006) 